\title{
System integration design in MEMS - A case study of micromachined load cell
}

\author{
SHISHIR KUMAR*, K P VENKATESH, S SAM BASKAR and \\ S P MADHAVI
}

CranesSci MEMS Laboratory, Department of Mechanical Engineering, Indian Institute of Science, Bangalore 560012

e-mail: shishir@mecheng.iisc.ernet.in

\begin{abstract}
One of the critical issues in large scale commercial exploitation of MEMS technology is its system integration. In MEMS, a system design approach requires integration of varied and disparate subsystems with one of a kind interface. The physical scales as well as the magnitude of signals of various subsystems vary widely. Known and proven integration techniques often lead to considerable loss in advantages the tiny MEMS sensors have to offer. Therefore, it becomes imperative to think of the entire system at the outset, at least in terms of the concept design. Such design entails various aspects of the system ranging from selection of material, transduction mechanism, structural configuration, interface electronics, and packaging. One way of handling this problem is the system-in-package approach that uses optimized technology for each function using the concurrent hybrid engineering approach. The main strength of this design approach is the fast time to prototype development. In the present work, we pursue this approach for a MEMS load cell to complete the process of system integration for high capacity load sensing. The system includes; a micromachined sensing gauge, interface electronics and a packaging module representing a system-in-package ready for end characterization. The various subsystems are presented in a modular stacked form using hybrid technologies. The micromachined sensing subsystem works on principles of piezo-resistive sensing and is fabricated using CMOS compatible processes. The structural configuration of the sensing layer is designed to reduce the offset, temperature drift, and residual stress effects of the piezo-resistive sensor. ANSYS simulations are carried out to study the effect of substrate coupling on sensor structure and its sensitivity. The load cell system has built-in electronics for signal conditioning, processing, and communication, taking into consideration the issues associated with resolution of minimum detectable signal. The packaged system represents a compact and low cost solution for high capacity load sensing in the category of compressive type load sensor.
\end{abstract}

Keywords. Piezo-resistance; force sensor; load cell; substrate coupling; ANSYS Multiphysics.

*For correspondence 


\section{Introduction}

System integration is characterized by any combination of more than one active electronic component of different functionality plus other devices like MEMS or non-MEMS based sensors or actuators assembled into a single standard package. Over the past few decades we have witnessed rapid advances towards the solutions to design issues in MEMS subsystems, whether it is a sensing or an actuating element design, fabrication process related, electronics interface, characterization, packaging etc. A typical design of an integrated transducer using MEMS technology requires proven procedure to be followed at each interface physics. The issue of integration in a single package gets more complex as the system integration process varies not only at a device level but also at application level. In most of the cases, the functions at each subsystem do not scale with IC/CMOS technology and in many cases their realization requires non-silicon materials and interface designs. Thus, the need for new integration solutions in MEMS technology is very much felt that combine single chip integration with hybrid assembly technology. This hybrid technology approach can be an optimized technology in itself and can be customized for each subsystem functions in a short development time and at lesser development cost.

System integration development needs to be carried out in a way that every subsystems and components needs an integrated approach of development. The interrelationship and interface physics at each component and subsystem level talks to overall system performance. An organization of various functional elements (components and subsystems) to build the basic architecture of the system defines the overall performance (Maarten Korsten \& Paul Regtien 2003). In order to fulfill the demands of MEMS technology in a system integrated form, various observations and design approaches can be derived from system in package technology. The system integration solution proposed here has been realized based on silicon technology with interconnects on ceramic substrate, and PCBs stacked with it for signal conditioning and processing features. We discuss design and development of components and subsystems such as sensing gauge interconnects, electronics interface, etc., which are essential for system design.

The basic design of the sensing gauge using silicon technology for load sensing can be found in (Elwenspoek et al 2000; Robert et al 2000; Remco Wiegerink et al 2000). However, the earlier work mentioned above do not address the issues of overall system design, and focused more on design and development of transduction element i.e. sensing gauge. In our present work, our main theme is to take the case of micromachined load cell and present a development approach of MEMS complete system. Moreover, we also propose different technique in terms of subsystem design (in this case sensing gauge), fabrication and packaging as compared to the existing work (Robert et al 2000), which needs to be accounted at the level of subsystem design, and their effects on overall system. Basically, load cell is a transducer which converts force into a measurable electrical signal. Based on measurement technique, these transducers can measure loads from micrograms to tones (Ernest Doebelin 2004). The compressive type load cells are widely used for measuring higher loads, which are typically made of metallic strain gauges as the sensing elements (Opperman 1985). One particular choice have been made earlier (Robert et al 2000; Elwenspoek et al 2000), using silicon as a material for spring element (sensing gauge) of the load cell and the microfabrication technique for its manufacturing. In the present work, we have made an attempt to explore the advantages of silicon technology (Elwenspoek et al 2000) while addressing various design considerations in making a complete system of MEMS-based load cell. There are number of factors that need to be considered at an early stage of the design process while proposing 
any architecture of the complete transducer system. For example, design of spring element for its linearity, ease of mounting and fabrication; overload protection, selectivity of response (load cell responding to forces along only one axis, while being insensitive to forces in other directions), response independent of the point of load application, and thermal effects on the sensing gauge. Typically, the relative importance given to any of these depends on the type and application of the particular transducer and moreover, these design considerations and parameters involved are not independent but have numerous interactions among subsystems. In the present work, we have focused on areas such as, (a) package design for direct contact type sensing stimulus for characterization of the device, (b) noise at electrical signal output, (c) noise due to mechanical loading and effect of unloaded silicon gauge on the final output, (d) microfabrication of all 4 resistors on the same chip to complete the resistance bridge circuit, and to avoid effect of residual stresses and TCR. We have also eliminated the need of any external SMD component for completion of bridge, with all 4 resistances fabricated on the same chip, (e) microfabrication using CMOS process for $\mathrm{SiO}_{2} \mathrm{RIE}$ to create sensing gauges. The interconnects have been made to keep all resistors ends open so as to examine each resistor individually, and, (f) use of two gauges simultaneously for loading to approximate half-bridge configuration, and hence, to increase sensitivity. The points mentioned from ( $a-f)$ above are necessary conditions for system integration, and design trade-offs of each component and subsystems and its interface with the next needs to be considered at the concept stage of system design. We present these processes of system integration and incorporated the design changes in detail by taking a case study of a MEMS sensor (Robert et al 2000; Elwenspoek et al 2000, Remco Wiegerink et al 2000) towards fulfillment of a MEMS-based integrated system.

\section{Proposed layout and operation}

In a typical load transducer system (compressive type), metallic or silicon spring elements can be used with elasticity or piezo-resistive property of the material (Opperman 1985; Robert et al 2000). While the previous work presents the possibilities of application of these materials or techniques for a load transducer system, we believe that there is a need to address all the design considerations and its integration to complete the whole system. For example, issues like on-chip temperature compensation without using any external resistors, completion of passive electronics on chip, active electronics in a single package, variation of output due to mounting of spring element on a silicon substrate and its effect on load calculation due to stresses in unloaded gauges. The force sensor presented in the present work is a compressive type load cell using polysilicon as a spring (sensing) element, which is deposited on patterned silicon dioxide grown on a silicon substrate. When the load is applied, the sensing element deforms and causes the change in resistance due to stresses and the piezo-resistive property change.

The proposed layout of the sensing element to be used in the load cell is shown in figure 1 . All the four gauges are deposited on the oxidized silicon substrate, where the design objective is to address issues like; completion of on-chip passive electronics without using any external resistors (SMD components), temperature compensation, reduction of offset voltage and its variations due to intrinsic stresses at fabrication level, and better sensitivity in the rated load capacity by using two active (load carrying) resistors. The other two gauges on the chip are being used for temperature compensation and to balance the bridge configuration. The fabrication of the proposed layout is carried through etching of deposited $\mathrm{SiO}_{2}$ while positioning the gauges at differential heights as shown in figure 1 . The fabrication steps in the 


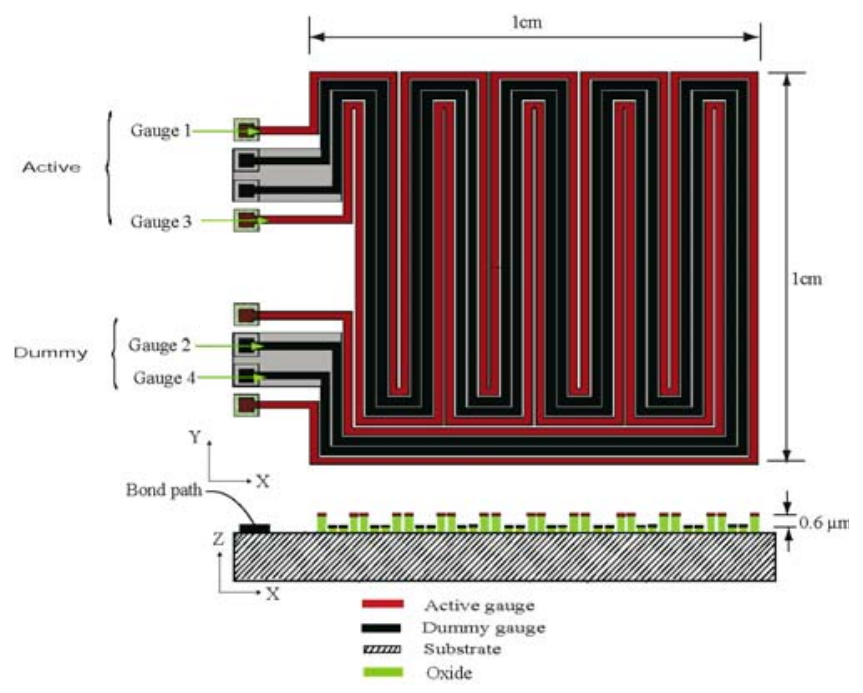

Figure 1. Layout of piezoresistive load cell.

present work are placed differently as in (Robert et al 2000) by using CMOS process. In the present work, the sensing part of the system basically consists of two pair of resistive gauges where polysilicon piezo-resitive property is used for sensing. The pair of active gauges made of ploysilion on etched $\mathrm{SiO}_{2}$ is used.

Other relevant design considerations presented in the proposed layout includes (a) effect of stresses in dummy gauges (unloaded) due to stresses in active gauge, (b) variation of resistance values on temperature. The solutions for both (a) and (b) can be proposed by the choice of layout and interconnects in such a way that the resistors form a voltage divider configuration with all resistors on chip with same TCR values and having same residual stress effects. In the succeeding section, we show that this interconnect allows to do simple calculations where the effect on the gauges gets cancelled out, however, based on FEM simulation, we strongly recommend that the effect of stress transfer on unloaded gauge needs to be accounted. This is an important consideration of any typical load cell design in order to show that it directly measures the forces, which is input on the axis of measurement.

\section{Design}

Piezo-resistance is a phenomenon referring to a change in resistance caused by the change in bulk resistivity $(\rho)$ of the material when subjected to mechanical stress. The resistance of a planar strip resistor as shown in figure 2a is $R=\rho(l /(w h))$; where $\rho$ is the resistivity and $l, w$, and $h$ are the length, width and thickness of the resistor, respectively. The change in resistance ' $R$ ' can be written in terms of strain and poison's ratio ' $v$ ' as:

$$
\frac{\Delta R}{R}=\frac{\Delta \rho}{\rho}+\varepsilon(1+2 v) .
$$

The relationship between the resistivity change $(\Delta \rho)$, current density and the stress components can be written as (Bao 2000):

$$
[\rho]=\rho_{o}[I]+\rho_{o}[\Delta],
$$

where $\Delta$ is a tensor of second rank determined by piezo-resistive coefficients, as $\Delta=[\pi]\{\sigma\}$. 
Using equation (1), (2) and the relationship between electric field and the resistivity, change in resistivity in different directions can be written as (neglecting the effect of shear stresses)

$$
\begin{aligned}
& \frac{\Delta \rho_{x x}}{\rho}=\pi_{l} \sigma_{x x}+\pi_{t}\left\{\sigma_{y y}+\sigma z z\right\} \\
& \frac{\Delta \rho_{y y}}{\rho}=\pi_{l} \sigma_{y y}+\pi_{t}\left\{\sigma_{x x}+\sigma z z\right\} \\
& \frac{\Delta \rho_{z z}}{\rho}=\pi_{l} \sigma_{z z}+\pi_{t}\left\{\sigma_{y y}+\sigma x x\right\} .
\end{aligned}
$$

Using equations (1) to (5), neglecting the resistance change due to geometry variation and if $\alpha$ is temperature coefficient of resistivity, we can write the relative change in resistance $(\Delta R / R)$ as:

$$
\frac{\Delta R}{R}=\pi_{1} \sigma_{1}+\pi_{t} \sigma_{t}+\alpha T
$$

where ' $\pi_{l}$ ' and ' $\pi_{t}$ ' are longitudinal and transverse piezo-resistive coefficients and are related gauge factors as (Robert et al 2000)

$$
\pi_{l}=\frac{G_{l}-2 G_{t} v}{E} \text { and } \pi_{t}=\frac{G_{t}(1-v)-G_{l} v}{E}
$$

where $E$ is youngs modulus and $G_{l}, G_{t}$ are lateral and traverse gauge factors of the polysilicon.

The above expression holds good for simple geometry of a piezo-resistive patch as shown in figure 2a and may not give an accurate results in structures like that of meander type as shown in figure $2 b$, where both the stresses and current density will be non-uniform in certain regions. As the length of the gauge along the direction of current flow (longitudinal) is higher than those in transverse directions (figure 1) the problem can be modelled as a set of planar strips running along its longitudinal axis.

From figure 3, we can observe that the gauges are subjected to stresses in $x, y$, and $z$ directions expressed as $\sigma_{x x}, \sigma_{y y}$ and $\sigma_{z z}$, respectively on application of load in $z$-direction. Stresses in the gauges can be expressed as local stresses (due to loading) and caused by material deformation (Timoshenko et al 1970). The effect of different stresses not contributing to resistance change but acting on the device is explained in (Robert et al 2000). In the present
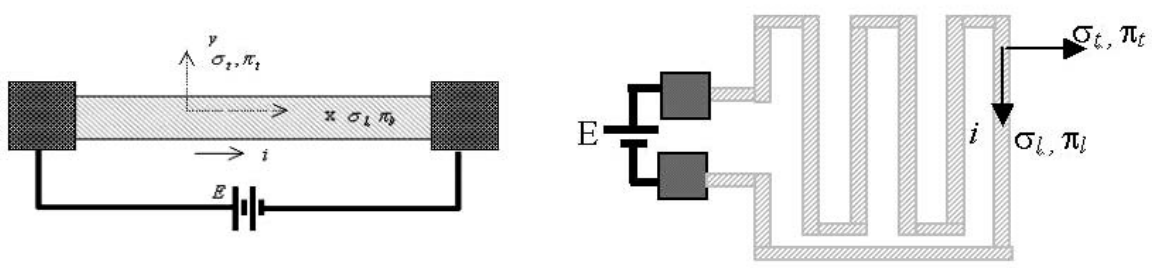

Figure 2. (a) Resistor in planar strip form. (b) Meander structure form of resistor. 


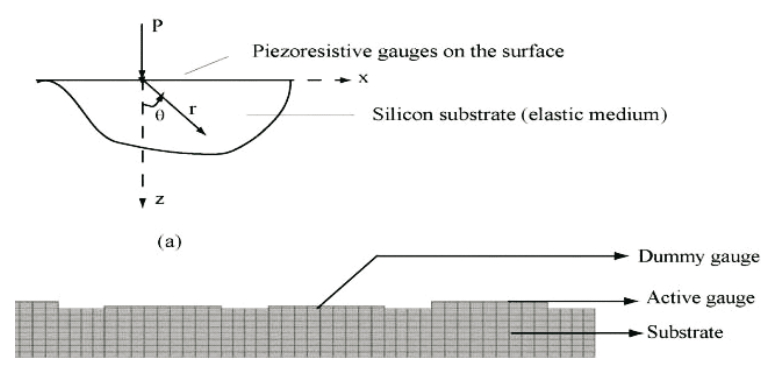

(b)

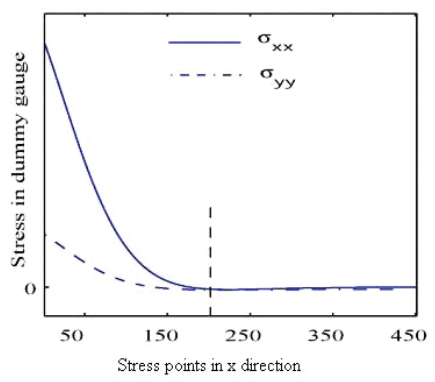

(c)

Figure 3. Substrate coupling effect.

work, we cover in detail the effect of local stresses on active and dummy gauges that cannot get cancelled out even with the choice of layout and interconnects. Here, we emphasize its contribution on the change in resistance measurement and below expressions follows the explanations. In figure $3 \mathrm{c}$ we can see that the placement of gauges even at larger gap may not help.

From equation (6), neglecting shear stresses in the structure, change in resistance in gauge 1 $\left(R_{1}\right)$ can be expressed as:

$$
\frac{\Delta R_{1}}{R}=\pi_{l}\left[\sigma_{x x}\left(\sigma_{z z}\right)\right]+\pi_{t}\left[\sigma_{y y}\left(\sigma_{z z}\right)+\sigma_{z z}\right]+\alpha T,
$$

where $\sigma_{x x}\left(\sigma_{z z}\right)$ represent stress in $x$-direction due to stress in $z$-direction (direction of application of load). Similarly, $\sigma_{y y}\left(\sigma_{z z}\right)$ corresponds to stress in $y$-direction due to stress in $z$ direction. As shown in figure 1, the gauge 1 and gauge 3 are being active to the applied load, together it contributes to change in resistance and can be expressed as:

$$
\frac{\Delta R_{1,3}}{R}=2 \pi_{l}\left[\sigma_{x x}^{1,3}\left(\sigma_{z z}\right)\right]+2 \pi_{t}\left[\sigma_{y y}^{1,3}\left(\sigma_{z z}\right)+\sigma_{z z}\right]+\alpha T .
$$

In a similar way, stresses in gauge 2 and gauge 4 (unloaded) can be expressed as:

$$
\frac{\Delta R_{2,4}}{R}=2 \pi_{l}\left[\sigma_{x x}^{2,4}\left(\sigma_{z z}\right)\right]+2 \pi_{t}\left[\sigma_{y y}^{2,4}\left(\sigma_{z z}\right)\right]+\alpha T
$$

where $\sigma_{z z}$ are the stress in active gauge.

We can express (9) and (10) by introducing constants $K_{1}=\frac{\sigma_{x x}^{1}}{\sigma_{z z}}, K_{2}=\frac{\sigma_{y y}^{1}}{\sigma_{z z}}, K_{3}=\frac{\sigma_{x x}^{2}}{\sigma_{z z}}$ and $K_{4}=\frac{\sigma_{x x}}{\sigma_{y y}^{2}}$. Thus,

$$
\begin{aligned}
& \frac{\Delta R_{1,3}}{R}=2 \pi_{l}\left[K_{l} \sigma_{z z}\right]+2 \pi_{t}\left[K_{2} \sigma_{z z}+\sigma_{z z}\right]+\alpha T \\
& \left.\frac{\Delta R_{2,4}}{R}=2 \pi_{l}\left[K_{3} \sigma_{z z}\right]+2 \pi_{t}\left[K_{4} \sigma_{z z}\right)\right]+\alpha T .
\end{aligned}
$$


Since, in the proposed layout we can create a voltage divider with active gauges $\left(R_{1}, R_{3}\right)$ and dummy gauges resistors $\left(R_{2}, R_{4}\right)$, change in resistance in a bridge configuration can be given as:

$$
\frac{R_{1,3}-R_{2,4}}{R}=2\left[\pi_{l}\left(K_{1}-K_{3}\right)+\pi_{t}\left(1+K_{2}-K_{4}\right)\right] \sigma_{z z}
$$

We can observe equation (13) in detail and conclude that change in resistance with an applied load and corresponding stress along one axis, $\sigma_{z z}=\frac{F}{W L}$, where $F$ is the applied force, $W$ is the width of the gauge and $L$ is the total length of the gauge, is independent of temperature variation.

$$
\frac{R_{1,3}-R_{2,4}}{R}=2 K \frac{F}{W L}
$$

where $K=\left[\pi_{l}\left(K_{1}-K_{3}\right)+\pi_{t}\left(1+K_{2}-K_{4}\right)\right]$. In an ideal case, as shown in (14) the value of ' $K$ ' should be exact material property, which can be represented by piezo-resistive coefficient values. However, through simulation studies it can be shown that in this case of discussion we cannot neglect the effect of stresses in other gauges. This leads to deviation in the value of ' $K$ ', that implies that we need to observe the magnitude of variation in change in resistance values with considerable effect due to $K_{1}-K_{3}$ and $1+K_{2}-K_{4}$ terms. In the succeeding section, we discuss in detail the effect of ' $K$ ' on calculation of resistance using FEM solution.

\subsection{Modelling}

Since, the stress pattern is constant along the axis, a cross section can be modelled for this particular problem, and we can examine the two dimensional behaviour of a large elastic medium for the loading as shown in figure 3. The analysis from (Timoshenko et al 1970) shows that at the point of application of the load, the stresses are large and follows a radial distribution pattern. We also know that the compression along one direction is coupled with expansions along other directions, which is characterized by the material property known as poisson's ratio. Considering this, we show in figure 4 the simulation results on a section of the sensing structure, the effect of normal stresses on the dummy gauges placed in close vicinity to the point of application of the load. We call this study as 'substrate coupling effect' on piezo-resistive sensing gauges acting as resistors and susceptible to variation in values due to stresses being transformed through the substrate on which it is placed. In the proposed measurement technique, we investigate this effect on the performance characteristics of the load cell and with this study we show the existing typical values of $K_{1}, K_{2}, K_{3}$ and $K_{4}$ due to the presence of stresses in all the gauges. This study is further justified from transducer design considerations point of view, as the design objective typically involves the isolation of any load, which can add to the response of the transducer other than it is designed for in any particular axis.

\subsection{Discussions}

Simulations are carried out in ANSYS to observe the stress pattern on different gauges. Figure 4 shows the geometric and stress analysis details, and relative change in resistance is calculated. Figure 4a shows the geometric details of fabricated device, and figure $4 \mathrm{~b}$ shows the results of 2D stress analysis. It is observed that stresses are also present in dummy gauges, 


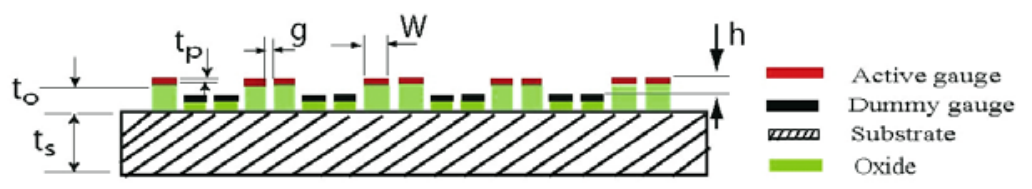

(a)

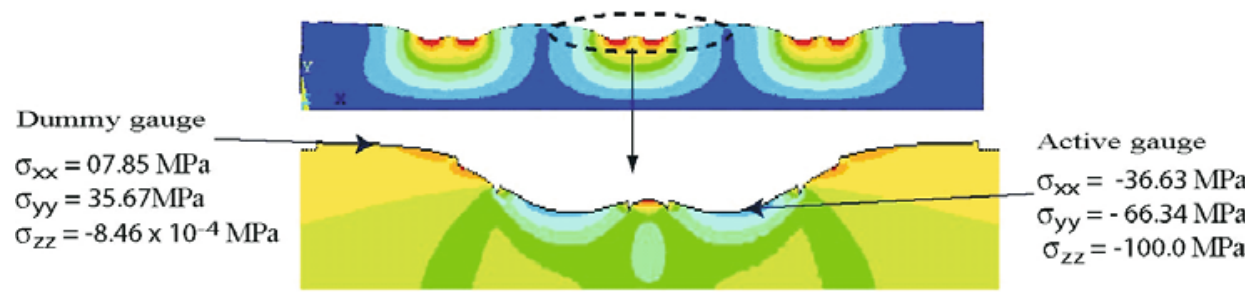

(b)

Geometric and Material details

\begin{tabular}{|c|c|c|c|c|c|c|c|c|}
\hline \multicolumn{6}{|c|}{ All dimensions are in $\mu \mathrm{m}$} & \multirow[b]{2}{*}{ Die size } & \multirow[b]{2}{*}{$\mathrm{E}(\mathrm{GPa})$} & \multirow[b]{2}{*}{$v$} \\
\hline$t_{s}$ & $t_{0}$ & $t_{p}$ & $\mathrm{~W}$ & $\mathrm{~g}$ & $\mathrm{~h}$ & & & \\
\hline 500 & 0.8 & 0.4 & 175 & 50 & 0.6 & $1 \mathrm{~cm} \times 1 \mathrm{~cm}$ & 166 & 0.22 \\
\hline
\end{tabular}

Figure 4. (a) Geometric details. (b) Stress distribution in active and dummy gauges.

which arises due to substrate coupling effect as discussed in previous section. Through simulation results it can be observed that the factor mentioned above $K_{1}-K_{3} \neq 0$ and the factor $\left(1+K_{2}-K_{4}\right) \neq 1$ and both the bracketed term carries almost finite values which is considerable for a typical load for example $K_{1}-K_{3} \sim 0.4448$ and $\left(1+K_{2}-K_{4}\right) \sim 2.0201$. This leads to the conclusion that the factor ' $K$ ' needs to be accounted in the final expression of the change in resistance. In this case the output voltage from the bridge is more than the expected values for the corresponding load as observed from equation (15) and shown in figure 4b.

As per the available fabrication process discussed in next section we have taken the values of $G_{l}$ and $G_{t}$ and corresponding values of $\pi_{l}$ and $\pi_{t}$ are calculated using equation (7) to estimate the final output voltage. Using $G_{l}=26, G_{t}=-6$ and the simulation results of $\frac{\Delta R_{1,3}}{R}=0.0047$ and $\frac{\Delta R_{2,4}}{R}=-0.001$, the output voltage $V_{\text {out }}$ is calculated as $13 \mathrm{mV}$, for a maximum load of $300 \mathrm{~kg}$ from equation (15). Forming two voltage dividers each containing one active and one dummy gauge can achieve the value of $\frac{\left(\Delta R_{1,3}-\Delta R_{2,4}\right)}{R}$ as shown in the figure 5a. Thus, the output voltage of the Wheatstone bridge formed is given by:

$$
\Delta V_{\text {out }}=\frac{1}{2} \frac{\Delta R_{1,3}-\Delta R_{2,4}}{R}=K \frac{F}{W L} V_{\text {in }} .
$$

Considering the factor of safety to be extremely high, we expect the output $V_{\text {out }} \sim 13 \mathrm{mV}$ for a load of $300 \mathrm{~kg}$ with a sensitivity of $8.6 \mu \mathrm{V} / \mathrm{V} / \mathrm{kg}$.

Typically, the common field of study for the characterization of material failure strength can be the strength of materials approach and the fracture mechanics approach. In both approaches, the failure strength is dependent on the relevant material properties. In this work, we have used the maximum principal stress theory and not the fracture mechanics approach to determine the failure strength of silicon. In the condition, where we have more information on microstructure of the thin film post fabrication, the fracture mechanics approach can be 


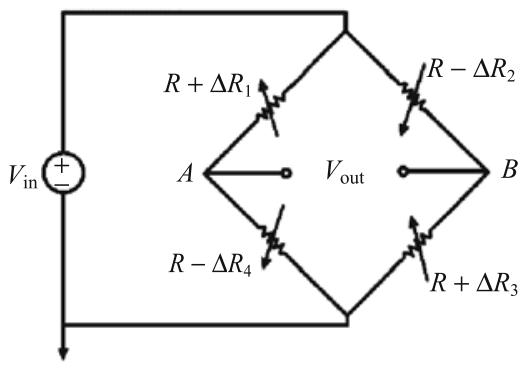

(a)

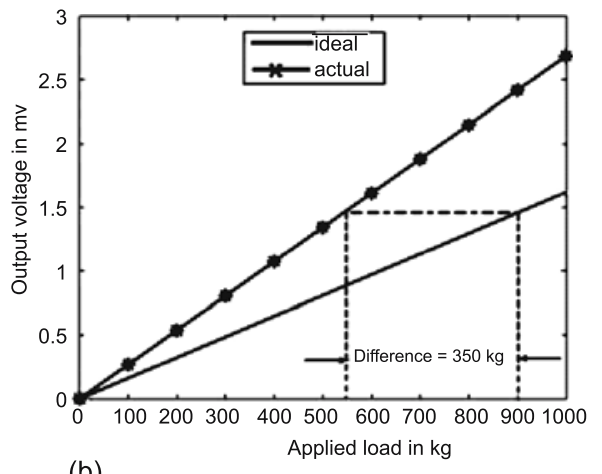

(b)

Figure 5. (a) Wheatstone bridge configuration. (b) Comparison between ideal and actual output.

used to arrive at more accurate results considering the stress intensity factor for the kind of loading that is required in this device.

\section{Microfabrication process}

The device presented here consists of four piezo-resistive sensing gauges; amongst which two are active and other two are passive. These are located at different heights, so that only active gauges are loaded while the passive gauges remain unloaded. For example, the required height difference (Robert et al 2000) involves etching of Si. But in the present case, the required height difference between the gauges is obtained by etching of $\mathrm{SiO}_{2}$ layer, and fabricated in CMOS foundry, followed by the standard process parameters.

The fabrication is started with initial oxidation of $P$-type $\langle 100\rangle$ silicon wafer for $0.8 \mu \mathrm{m}$ and patterned for the same thickness. Further oxidation is done for $0.2 \mu \mathrm{m}$ thickness to maintain the height difference of $0.6 \mu$ between the pair of active and passive gauges. Polysilicon layer of $0.4 \mu$ thickness is deposited on the surface of the formed oxide using LPCVD at $610-625^{\circ} \mathrm{C}$. The desired electrical and piezo-resistive characteristics like sheet resistance of $185-205 /$ square, low temperature coefficient of resistance of $-0.02 \%$ to $0.02 \%$ and piezoresistive strain coefficients of 24-28 can be achieved by implanting $1.5-2.5 \times 10^{19} \mathrm{~cm}^{-3}$ of boron atoms and subsequent annealing for 30 minutes at $1050^{\circ} \mathrm{C}$ (French 1989; Manjula et al 2006; Le Berre et al 1995; Mosser et al 1991). Further, the layer is patterned and reactive ion etching is done as per the required feature size of sensing gauges. It is necessary to protect the gauges and the underlying oxide layer during the process of native oxide removal. Since the selectivity of enchants used for removing native oxide and silicon nitride is different, it can be used as passivating material to protect the structure. Thus, the structure is passivated by depositing (PECVD) silicon nitride for $1000 \mathrm{~A}^{\circ}$. Realization of the device is completed by subsequent process of contact opening, Aluminum metallization and removal of passivation layer using four masks and dry etching process.

Figure 6 shows the steps followed for the fabrication of the load cell device. Followed by dicing the device is attached on the ceramic board where it gets interconnected using hybrid technology and forms whetstone bridge configuration.

Making the bridge configuration is a part of the packaging process, which involves three major processes, interconnection on the ceramic board, device attachment and wire bonding. 

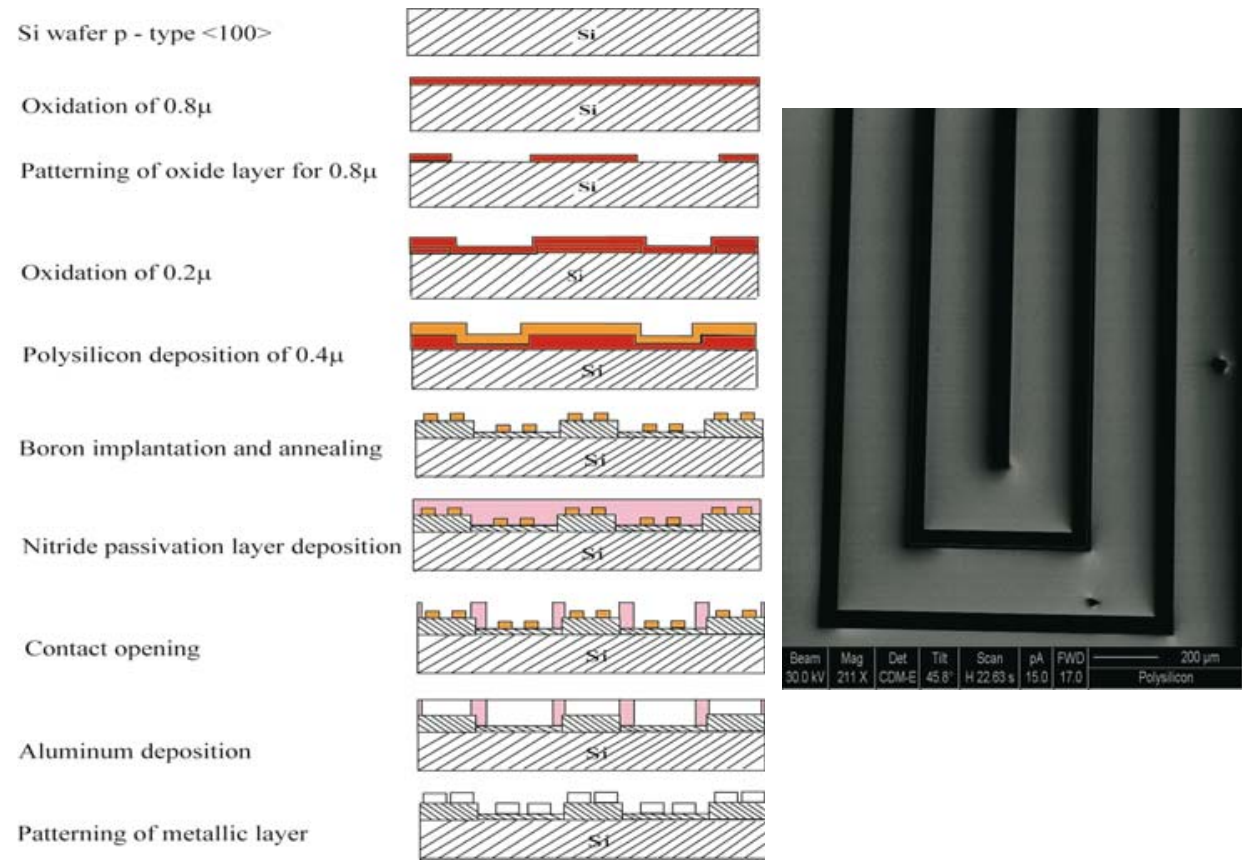

Figure 6. (a) Steps followed for the fabrication of the load cell, (b) image of the fabricated gauge (only part of the full gauge is shown).

Attachment of the device on the ceramic board is obtained by means of gluing which provides mechanical support. Reichl (1991) has shown the advantage of epoxy gluing and properties of ceramic board in terms of low stress transfer to the device, which makes it a good choice of bonding method and material for device attachment. Silver palladium interconnect tracks are made on the ceramic board using screen-printing technology. Wire bonding between the bond pads of the device and interconnects on the ceramic board completes the bridge configuration and provides electrical connectivity for characterization.
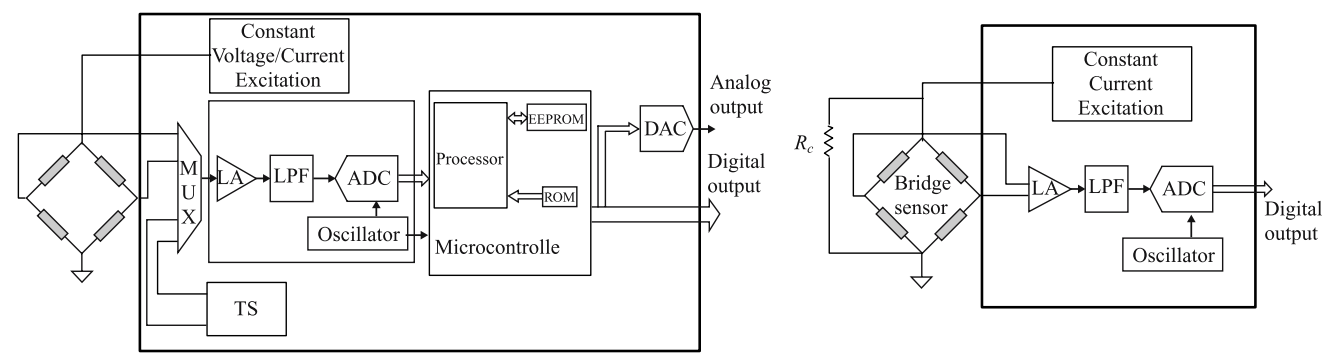

Figure 7. (a) Block diagram of typical signal conditioning unit. (b) Signal conditioning for the load cell. 


\section{Electronics interface}

The full load output voltage of any piezo-resistive MEMS based sensors, are in the range of millivolts, and may suffer from noise and other non-idealities, which makes it difficult to measure small changes in resistance (Bao 2005). Process variations and thermal effects lead to the offset voltage, which reduces the span of the sensor. A basic signal conditioning circuit should address all these non-idealities.

A typical block diagram of the signal conditioning unit (SCU) is shown in figure 7a. The differential signal from the piezo-resistive bridge sensor is amplified by the low noise, high CMRR and high input impedance instrumentation amplifier (IA) (Ramon Pallas 2001). Compensation for the non-idealities can be achieved either digitally or using analog electronics. Many signal conditioning ICs are available commercially (Texas Instruments 2004; Maxim 2005) which provide solutions towards signal conditioning of sensors working on resistive sensing technique and compensates for the non-idealities to an extent.

In the present case study, the load cell carries a resistance value of $\sim 90 \mathrm{k} \Omega$ with the sensitivity of $50 \mu \mathrm{V} / \mathrm{kg}$. Considering, noise at the sensor level only for the load cell, the minimum detectable signal of the sensor is $\sim 17$ grams (Richard et al 1988). The resolution of the sensor can be improved with the instrumentation amplifier, however, high amplifier gain settings produce a high output noise and the integration needs to be done using higher ADC resolution. Typically, resistive sensing based load transducers require count of 1:30,000 to have a weight resolution of 10 grams, and demands at least 20 bit ADC (Colm Slattery \& Mariah Nie 2005) and in some cases the digital output are achieved for further processing in the communication. The signal conditioning for the load cell implemented on the PCB is shown in figure $7 \mathrm{~b}$.

\section{Packaging for characterization}

Packaging of MEMS devices is complex in the sense that in some circumstances the prime objective is to provide protection from the environment while in others the priority is to allow access to the environment to measure or affect the desired physical parameters. Therefore, testing MEMS packages for the same device with standard procedures might not always be possible, especially when the device is being used for different applications. The design of experiments on a MEMS device requires detailing from the material characteristics point of view to the signal extraction. This includes, issues from fabrication and the technique of input for the measurand to the device. While silicon being a brittle material, moderate stress levels could result in failure such as cracking and fracture in the die (Scott 1998). In addition, waferprocessing steps such as dicing could induce defects in the silicon die. Due to surface and edge defects present in typical silicon available for die testing, when subjected to stress loading, there exists a high potential that the micro cracks would propagate and result in fracture of the die. As such, the die crack or fracture can commonly be resulted during assembly process too (Desmond et al 2004). This particular fact becomes very prominent in the type of device that we present here and hence the design for experiments involves various issues to be considered for a direct contact of the measurand on the silicon die. We present various issues that we came across as shown in figure $8 \mathrm{~A}$ for this particular device based on our first pass of experimentation on the prototype shown in figure $8 \mathrm{C}$.

There are mainly two objectives in the design of the proposed package: one is to provide a method by which homogeneous compressive load gets transferred to the die and second 


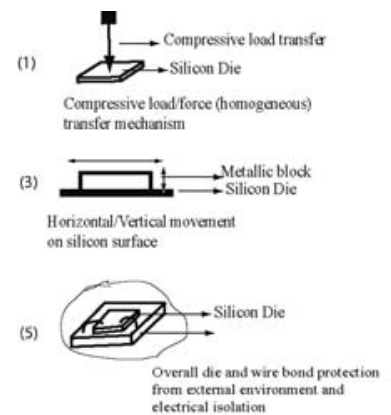

B

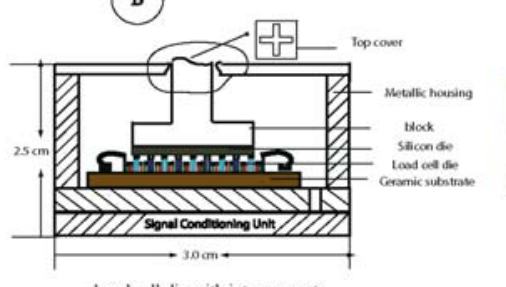

Load cell die with interconnects
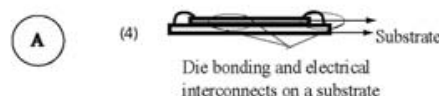

(๘)

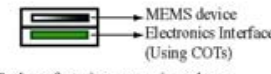

Padage featuring system in package
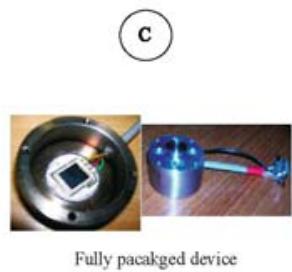

Fully pacakged device

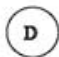

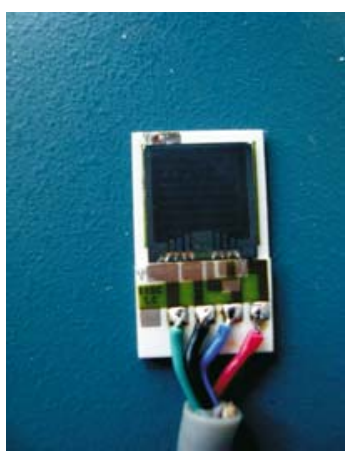

Figure 8. (a) Load cell assembly gauge and, (b) sensing gauge with interconnects.

is to differentiate and partition various factors that affect the die strength for the method adopted for the loading. Some of the typical considerations while designing this package is shown in figure $8 \mathrm{~A}$, where the key points from (1) to (6) are self-explanatory and to be considered for the brittle material like silicon when used in MEMS device (Desmond et al 2004; Robert et al 2000). Some of them being, the local stress concentrations due to metalsilicon surface contact, lateral-vertical movement on the die surface, electrical interconnects, signal conditioning and isolation from electronic interferences. The issues are described in the figure 8 and the proposed solution by various means is described in the figure $8 \mathrm{D}$.

\section{Conclusion}

A load cell working on resistive sensing (piezo-resistive effect) using polysilicon as a sensing material is designed, analysed and fabricated towards completion of a system integration process. Equal emphasis is given on the work involving design of an essential element of the load cell (sensing gauge) to the packaging of the device for compressive load applications. An approach is proposed to build a system in a package ready for characterization, and represented as prototype of a MEMS transducer system.

The particular MEMS device is designed for higher load applications in the range of $5 \mathrm{kN}$ with in-built interface electronics for signal conditioning. The method proposed here can be useful in the efforts of system integration based work in MEMS technology. The approach can be found suitable to expedite the processes of technology demonstration and advantageous in concept building for product development. At each subsystem level, the design of the present 
form factor may provide further levels of developmental work in the area of integration of electronics with this MEMS sensor.

\section{References}

Bao Min-Hang 2000, Piezo-resistive sensing, Handbook of Sensors and Actuators Volume 8 Elsevier Bao Min-Hang 2005, Piezo-resistive sensing, Analysis and design principles of MEMS devices. Elsevier

Colm Slattery, Mariah Nie 2005 A reference design for high performance low cost weigh scales. Analog Dialogue 39: 1-6

Desmond Y R, Chong W E, Lee B K Lim, John, Pang H L, Low T H 2004 Mechanical characterization in failure strength of silicon dice, IEEE Inter. Society Conference on Thermal Phenomena 203-209

Elwenspoek M, Wiegerink R 2000 Force and Pressure sensors, Mechanical Microsensors. Springer

Ernest O, Doebelin 2004 Force, torque, and shaft power measurement, Measurement systems applications and design, $5^{\text {th }}$ Edition, (New Delhi: Tata McGraw-Hill)

French P J, Evans 1989 Piezo-resistance in polysilicon and its applications to strain gauges. Solid-state Electronics 32(1): 1-10

Le Berre M, Kleimann P, Semmache B, Barbier D, Pinard P 1995 Electrical and piezo-resistive characterization of boron doped LPCVD polycrystalline silicon under rapid thermal annealing. Transducers '95 - EUROSENSOR IX 76-79

Maarten Korsten, Paul Regtien 2003 Systematic and computer-assisted design of measurement systems. Measurement 33: 145-156

Madou M 1997 Fundamentals of Microfabrication, Florida, CRC Press

Manjula S Raman, Teweldabhran Kitle, Enakshi Bhattacharya, Bhat K N 2006 Physical model for the resistivity and temperature coefficient of resistivity in heavily doped polysilicon. IEEE Transactions on Electron Devices 53(8): 1885-1892

Maxim 2005 User Guide MAX1464

Mosser J, Suski J Goss, Obermeier E 1991 Piezo-resistive pressure sensors based on polycrystalline silicon. Sensors and Actuators A28: 113-132

Opperman K 1985 A new force sensor with metal measuring grid transverse to the line of force. Sensors and Actuators 7: 223-232

Ramon Pallas-Areny, John G Webster 2001 Chapter 3, Sensors and signal conditioning, John Wiley

Reichl H 1991 Packaging and Interconnection of sensors. Sensors and Actuators A25-27: 63-71

Richard R Spencer, Bruce M Fleischer, Philip W Barth and James B Angell 1998 A theoretical study of transducer noise in piezo-resistive and capacitive silicon pressure sensors. IEEE Trans. on Electron Devices 35(8): 1289-1298

Robert A F Zwijze, Remco J Wiegerink, Gijs J M Krijnen, Theo S J Lammerink, Miko Elwenspoek 2000 Low-cost piezo-resistive silicon load cell independent of force distribution J. Micromechanics and Microengineering 10: 200-203

Scott F Popelar 1998 An investigation into the fracture of silicon die used in flip chip Applications. International symposium on Advanced Materials 41-48

Texas Instruments 2004 PGA309 Voltage output programmable sensor conditioner, User Guide

Timoshenko S P, Goodier J N 1970 Theory of elasticity, (USA: McGraw-Hill International)

Wiegerink R J, Zwijze A F, Mateman R, Krijnen G J M, Lammerink T S J, Elwenspoe M C 2000 Quai-monolithic silicon load cell for loads up to $1000 \mathrm{~kg}$ with insensitivity to non-homogeneous load distributions. Sensors and Actuators 80: 189-196 